\title{
Schlaffe Jungs sind Diabetes-Kandidaten
}

\section{Hier steht eine Anzeige.}

\section{Niedrige Ausdauerleistung und geringe Muskel- kraft bei jungen Männern im Alter von 18 Jahren erhöhen das Langzeitrisiko für Typ-2-Diabetes.}

- Übergewicht und sitzende Lebensweise gelten als wichtige Risikofaktoren für Typ-2-Diabetes. Geringe körperliche Aktivität und mangelnde Fitness sind zwar als weitere Risiken anerkannt, es fehlten bisher aber größere Langzeituntersuchungen, in denen die Fitness jüngerer Menschen objektiv gemessen und die Diabetesinzidenz im Verlauf der folgenden Jahrzehnte bestimmt worden wäre.

Für eine Studie wurden nun 1.534.425 schwedische Wehrdienstpflichtige der Jahre 1969-1997 ohne vorbestehenden Diabetes mellitus betrachtet - das sind mehr als 97\% aller 18-Jährigen dieser Alterskohorte. Um die Ausdauerleistungsfähigkeit zu bestimmen, wurden die maximale Sauerstoffaufnahme am Ergometer erhoben und die Muskelkraft in Beinen, Armen und Händen gemessen. Die Diabeteserkrankungen wurden bis 2012 aus dem schwedischen Register entnommen.

Über vier Jahrzehnte wurden 34.008 Diabetesfälle entdeckt. Im niedrigsten Terzil von Ausdauer und Muskelkraft lag die kumulative Inzidenz von Typ-2-Diabetes höher als im höchsten Terzil, und zwar um 0,22 Prozentpunkte nach 20 Jahren, um 0,76 Punkte nach 30 und um 3,97 Punkte nach 40 Jahren. Insgesamt war die schlechtere Fitness mit einem dreifach erhöhten Diabetesrisiko vergesellschaftet, auch bei Personen mit normalem BMI und unabhängig von Familienanamnese und sozioökonomischen Einflüssen.

- Crump C, Sundquist J, Winkleby MA et al. Physical fitness among Swedish military conscripts and long-term risk for type 2 diabetes mellitus. Ann Intern Med. 2016;164:577-84

\section{KOMMENTAR}

Es handelt sich um eine umfangreiche Untersuchung praktisch aller jungen Männer in Schweden. Man stützte sich nicht auf unzuverlässige Befragungen der Probanden, sondern auf objektive Messungen der körperlichen Fitness. Die Besonderheit der Daten besteht darin, dass geringe Ausdauerleistungsfähigkeit schon im Alter von 18 Jahren das Diabetesrisiko über die folgenden vier Jahrzehnte erhöht. Es gibt zwar keine Verlaufsuntersuchungen zur körperlichen Fitness und zum Körpergewicht nach der anfänglichen Wehrpflichtuntersuchung, doch belegen die Ausgangsdaten überzeugend, dass körperliche Fitness im Jugendalter langfristig bedeutende gesundheitliche Vorteile hat - auch bei Personen, die in der Jugend nicht übergewichtig und adipös sind.

Prof. Dr. med. H. Holzgreve

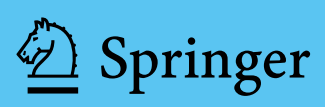

